\title{
Probability prediction of tensile strength with acoustic emission count of a glass fiber reinforced polyamide
}

\author{
Mohamed Makki Mhalla ${ }^{1, *}$, Ahmed Bahloul ${ }^{1,}$, and Chokri Bouraoui \\ Laboratoire de Mécanique de Sousse, Ecole Nationale d'Ingénieurs de Sousse Université de Sousse, Bp.264 Erriadh, 4023 Sousse, \\ Tunisie
}

Received: 21 June 2016 / Accepted: 10 February 2018

\begin{abstract}
The aim of this paper is to develop a probabilistic approach for predicting the tensile strength behavior of a glass fiber reinforced polyamide. In the present study, the reliability of tensile strength is proposed based on the developed mathematical models, in which three factors with three levels are implemented. Glass fiber content, temperature and strain rate are chosen as the main input parameters in this study. The tensile strength is considered as output response which is evaluated through experimental tests. The "Strength-Load" method with Monte Carlo simulation is implemented for computing the tensile strength reliability. The proposed approach leads to predict useful the tensile strength behavior for different parameters. In addition, a sensitivity analysis of some input parameters on the reliability is discussed. This method has been also used to analyze and discuss the influence of the dispersions of the glass fiber content and the temperature of a glass fiber reinforced polyamide.
\end{abstract}

Keywords: Reliability approach / response surface methodology / thermoplastic composites / Monte-Carlo simulation

\section{Introduction}

Due to their high specific strength and stiffness, glass fiber reinforced polyamides have been increasingly used in many applications these last decades, such as: stressed functional automotive parts (fuel injection rails, steering column switches) and safety parts (sports and leisure). Injection molding process is considered as the most conventional methods used for processing fiber reinforced thermoplastics compounds. It improves the mechanical properties over the unreinforced ones [1]. However, voids are always present in these injected materials in which cracks can be initiated and propagated in one of three regions: the matrix, the fiber or the fiber/matrix interface [2]. In this context, several works have dealt with the mechanical properties of thermoplastic composites containing short fibers. These properties result from a combination of the fiber, the matrix properties and the ability to transfer stresses across the fiber/matrix interface, but it also depends on many variables such as fiber ratio, diameter, length, orientation and the strain rate which are of prime importance to the final properties of the thermoplastic composites [3,4].

\footnotetext{
* e-mail: mhallamaki@yahoo.fr
}

Behavior evolution of composite materials is phenomena affected by high uncertainties where the deterministic approach fails to estimate exactly the damage fracture. In this area, reliability approaches become more and more considered as an engineering design in industrial application $[5,6]$. However, few studies have dealt with the case of reliability approach through composite materials $[7,8]$. Xueyong et al. [7] identified the uncertainties in composite material properties based on reliability optimization. They tacked into account the scatterings and the significant dispersions related to the geometrical and material parameters. The Response Surface Methodology is applied by Zhigang et al. [8] to investigate the reliability and to evaluate the risk of failure for complex structures such as a $2.5 \mathrm{D} / \mathrm{SiC}$ composite.

The objectives of the present paper consist in:

(i) the response surface methodology (RSM) coupled with experimental tests is used to investigate the effect and the interaction between the different factors (glass fiber content, temperature and strain rate) on the tensile strength of a glass fiber reinforced polyamide;

(ii) developing a probabilistic approach for evaluating the tensile strength reliability of a glass fiber reinforced polyamide by taking into account the dispersions of the glass fiber content and the temperature. The 'Strength-Load' method coupled with the Monte Carlo simulation (MCS) is implemented for computing the reliability of our material. 


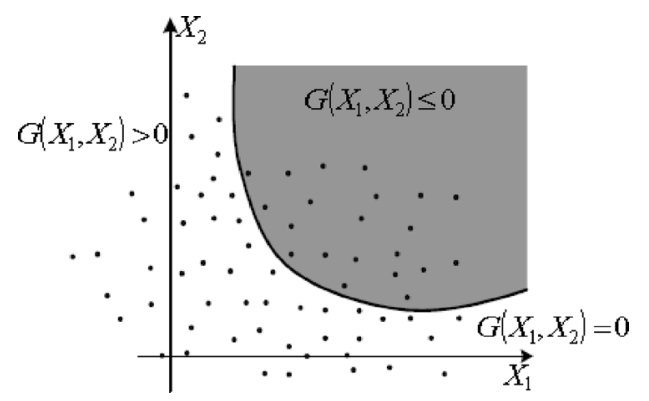

Fig. 1. Probability density function of $G(x)$ [12].

\section{Background of the Monte Carlo method}

To compute the reliability, several numerical methods can be used $[9,10]$, such as: (i) analytical resolution, (ii) approximate computational methods (first-order reliability method: FORM and second-order reliability method: SORM) and (iii) MCS.

The Strength-Load approach is considered to be one of the most reliably computational methods to evaluate the risk $[11,12]$.

To compute the reliability, one considers a vector of random variables $\{X\}$ representing uncertain structural quantities. Let xi be an element of the random vector $\{X\}$, with a probability density function $f_{X i}(X i)$. A performance function $G(\{X\})$, separating the security and the failure fields is written as follows:

$$
G(\{X\})=S(\{X\})-L(\{X\}),
$$

where $G(\{X\})=0$ is the limit state function, $S(\{X\})$ is the strength function and $L(\{X\})$ is the load function [12]. In that case, if the inequality $G(\{X\})>0$ is satisfied, this indicates a structural safety condition. In the opposite case, if $G(\{X\})<0$, this means a failure of such a structure (Fig. 1). $(G(\{x\})<0)$ is given by:

$$
\begin{gathered}
P_{f} \\
P_{f}=\operatorname{Pr}\left(G\left(x_{i}\right) \leq 0\right)=\int_{G\left(x_{i} \leq 0\right)} f_{\{X\}}\left(x_{i}\right) d x_{1} \ldots d x_{n}
\end{gathered}
$$

with $f_{X i}(X i)$ is the joint density function of $G(\{x\})$.

The failure probability is used to quantify risks that the behaviors of the fracture exceed a given criterion. To compute the failure probability $P_{f}$ is very difficult with analytical method this is due to the difficulty to know $f_{X i}(X i)$. For this reason, we can use the MCS: which is widely used in the case of high number of random variables and also when $f_{X i}(X i)$ is practically difficult to find. Using several random sampling, the Monte Carlo method aims to simulate a high number of load and strength values according to their PDF. For a total number of simulation $\mathrm{N}$, all events are represented by the computed values of $G(\{x\})$. It is well established that the failure event frequency, defined by $G(\{x\})<0$, extends towards the failure probability $P_{f}$ when $N \rightarrow+\infty$ [13]. Generally $N$ is taken equal to $10^{4}$, it is an acceptable computational cost especially in the case of explicit function [14].
The failure probability $P_{f}$ is then expressed as follows:

$$
P_{f}=\underset{\mathrm{N} \rightarrow \infty}{\lim } \frac{\text { Number of failure events }(G(\{x\})<0)}{N} .
$$

Finally, the reliability $R$ is given by the following relationship:

$$
R=1-P_{f}
$$

In this paper, we used the MCS to compute the reliability but an explicit relationship of the limit state function $G(\{x\})$ is needed, for this reason a response surface methodology [15] is performed to determinate the limit state function $G(\{x\})$.

\section{Experimental work}

\subsection{Design}

In this study, experiments were designed based on the experimental work given by Mouhmid et al. [16,17]. The main objective of the factorial experiments consists in studying the relationship between the different parameter levels and the response as a dependent variable. This approach helps to understand better how the change in the levels of each parameter can affect the response. The design required 27 experiments with 3 levels. The design was analyzed and generated using MINITAB 16.0 statistical package. Temperature, glass fiber content and strain rate are chosen as the main input parameters in this study. Table 1 shows the factors and their levels in coded and actual values.

\subsection{Experimental procedure}

The material used in the present study was short glass fiber reinforced polyamide $\mathrm{P} 66$ with $2 \mathrm{~mm}$ of total thickness. The length $(L)$ and width $(W)$ of plate are 250 and $25 \mathrm{~mm}$, respectively. The tensile tests were carried out with an Instron machine $(10 \mathrm{kN})$ equipped with a temperature controlled chamber. The compounds were molded with a molding machine with a capacity of 80 tons. Tests were carried out at different parameters such as: the glass fiber content is selected to be between 0 and $30 \%$, temperature is considered to be between 20 and $80^{\circ} \mathrm{C}$ and strain rate ranges from 1 to $49 \mathrm{~mm} / \mathrm{min}$.

The experiments have been carried out according to the designed experimentation as illustrated in Table 2.

\subsection{Reliability assessment}

Reliability methods have been established to take into account the uncertainties involved in the analysis of an engineering problem. In this approach, the Response Surface Method (RSM) can be used to evaluate the reliability. The dispersions taken into account in the present study are: (i) the Glass fiber content, (ii) the temperature. 
Table 1. Experimental parameters and their levels.

\begin{tabular}{lllll}
\hline Parameters & Notation & $\begin{array}{l}\text { Levels } \\
-1\end{array}$ & 0 & +1 \\
\hline$(\mathrm{mm} / \mathrm{s}) \dot{\varepsilon}$ & $A$ & 1 & 25 & 49 \\
$T\left({ }^{\circ}\right)$ & $B$ & 20 & 50 & 80 \\
$v_{f}(\%)$ & $C$ & 0 & 15 & 30 \\
\hline
\end{tabular}

Table 2. Experimental results.

\begin{tabular}{|c|c|c|c|c|}
\hline$N^{\circ}$ essai & $A$ & $B$ & $C$ & Tensile strength \\
\hline 1 & -1 & -1 & -1 & 65 \\
\hline 2 & -1 & -1 & 0 & 78 \\
\hline 3 & -1 & -1 & +1 & 85 \\
\hline 4 & -1 & 0 & -1 & 57 \\
\hline 5 & -1 & 0 & 0 & 75 \\
\hline 6 & -1 & 0 & +1 & 82 \\
\hline 7 & -1 & +1 & -1 & 48 \\
\hline 8 & -1 & +1 & 0 & 65 \\
\hline 9 & -1 & +1 & +1 & 74 \\
\hline 10 & 0 & -1 & -1 & 70 \\
\hline 11 & 0 & -1 & 0 & 80 \\
\hline 12 & 0 & -1 & +1 & 90 \\
\hline 13 & 0 & 0 & -1 & 59 \\
\hline 14 & 0 & 0 & 0 & 78 \\
\hline 15 & 0 & 0 & +1 & 88 \\
\hline 16 & 0 & +1 & -1 & 49 \\
\hline 17 & 0 & +1 & 0 & 68 \\
\hline 18 & 0 & +1 & +1 & 78 \\
\hline 19 & +1 & -1 & -1 & 73 \\
\hline 20 & +1 & -1 & 0 & 83 \\
\hline 21 & +1 & -1 & +1 & 95 \\
\hline 22 & +1 & 0 & -1 & 60 \\
\hline 23 & +1 & 0 & 0 & 79 \\
\hline 24 & +1 & 0 & +1 & 90 \\
\hline 25 & +1 & +1 & -1 & 49 \\
\hline 26 & +1 & +1 & 0 & 69 \\
\hline 27 & +1 & +1 & +1 & 80 \\
\hline
\end{tabular}

The general procedure used for computing the tensile strength reliability of the short glass fiber reinforced polyamide P66 is detailed trough the following steps:

Step1: Evaluation of tensile strength using the design of experiments

In this step, the design of experiments based on full factorial design is implemented. The glass fiber content, temperature and strain rate are chosen as the main input parameters. The MINITAB statistical package was used for predicting the effect and the interaction of the input parameters on the tensile strength.

Step2: Building the explicit limit state functions $G(\{x\})$ based on RSM
The computation of failure probability $\mathrm{P}_{\mathrm{f}}$ in equation (2) is not easy. For this reason, MCS is developed. An explicit limit state function $G$ depending on different random parameters based on the surface method is then needed. The Design of Experiments based on a full factorial design is used to build an explicit relationship of the basic safety margin, which links the tensile strength as a function of glass fiber content, temperature and strain rate using the response surface method.

Step3: Reliability updating assessment

In this work, the MCS method is used to calculate the reliability of a short glass fiber reinforced polyamide based on the explicit relationship developed in step 2. The glass fiber content and temperature with their corresponding dispersions are taken into account in the computing of the reliability. The different steps are detailed in the flowchart as shown in Figure 2.

\section{Results and discussion}

\subsection{Development of mathematical models}

The response surface method coupled with the experimental results is implemented for predicting the mathematical relationships of the tensile strength and the as a function of the glass fiber content, temperature and strain rate.

The analytical expression, obtained from analyzing the influences of the various dominant parameters on the tensile strength is given by:

$$
\begin{aligned}
\text { Tensile strength } & =66.82+0.2155 \dot{\varepsilon}-0.0523 T+0.965 v_{f} \\
& -0.0009 \dot{\varepsilon} \times \dot{\varepsilon}-0.0024 T \times T-0.0133 v_{f} \\
& \times v_{f}-0.0015 \dot{\varepsilon} \times T+0.0018 \dot{\varepsilon} \times v_{f} \\
& +0.0048 T \times v_{f} .
\end{aligned}
$$

In order to validate and check the accuracy of the constructed RS model, some points are generated. Figure 3 shows the verification point's results obtained using Experiment and RS models. The high value of $R$-squared (superior to $98.8 \%$ ) indicated that the model was highly reliable in predicting the tensile strength. The results of the RS models are in a good correlation with experimental results and provide accurate and satisfactory results.

\subsection{Effect and sensitivity analysis of input factors}

The effects of strain rate, temperature and glass fiber content on the tensile strength are illustrated in Figure 4.

It is observed that the temperature was found as a significant parameter in which an increase in the temperature value leading to decrease the tensile strength. However, an increasing of the strain rate and the glass fiber content leading to increase in the tensile strength.

The obtained results are used to evaluate the percentage contribution of each input factor as shown in Figure 5. Additional, the Pareto chart indicates main and interaction effects considered statistically significant. As apparent, the glass fiber content has the strongest effect of $66 \%$ on the tensile strength, and the temperature contributes $25 \%$. 


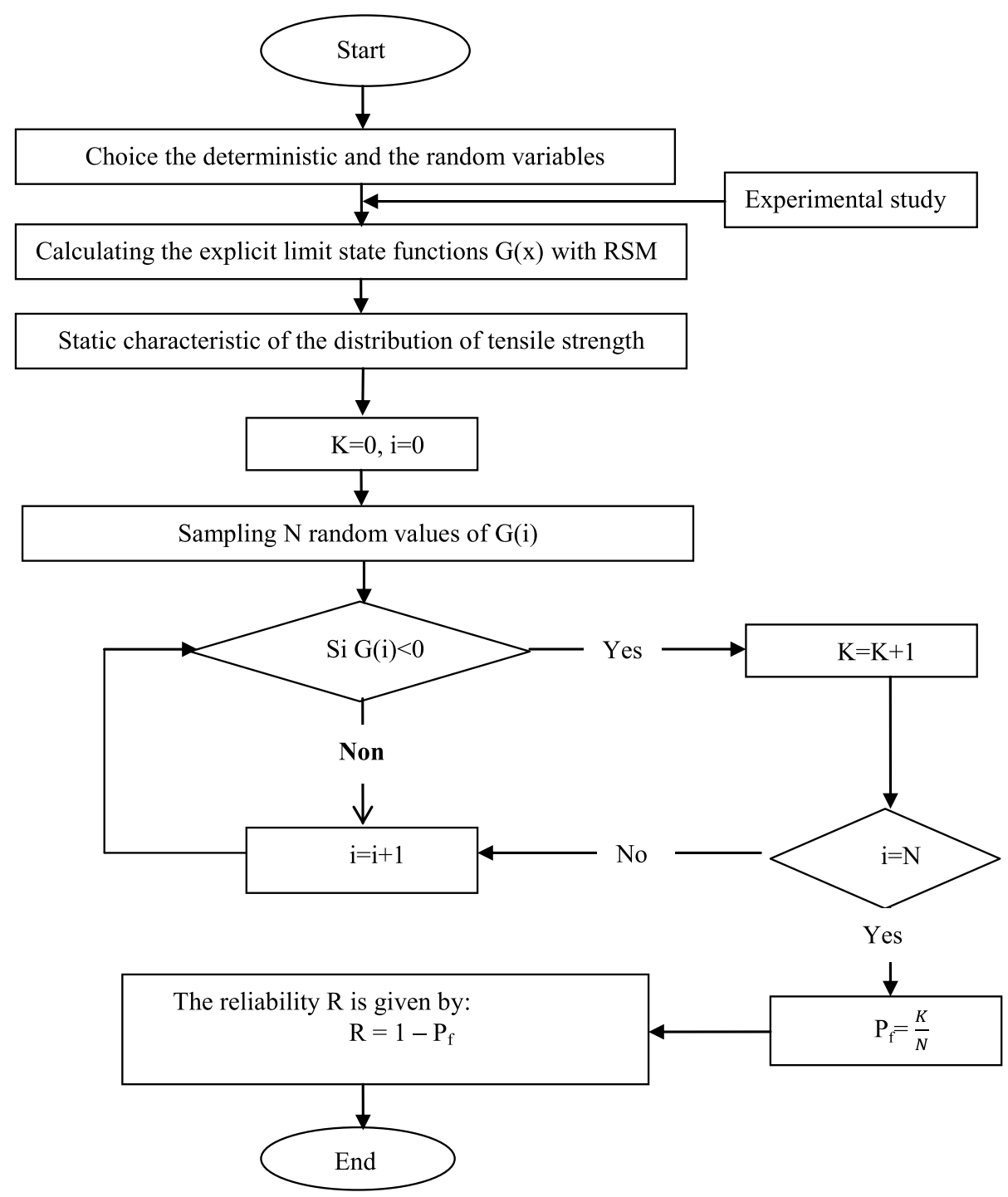

Fig. 2. Flow chart for the reliability computation.

From this analysis, it is confirmed that the effect of the strain rate was trivial, with a percentage contribution equals to $3.5 \%$ on the tensile strength. This observation is in good agreement with the previous results [18], in which they have found that the effect of strain on the strength tensile was negligible on the glass fiber reinforced polyamide composites.

The results show a low interaction between fiber content and the other parameters were observed. This observation is coherent with previous studies [19].

In this study, an analytical model is developed to predict the tensile strength of fiber glass reinforced polyamide using Response Surface Methodology.

\subsection{Reliability analysis}

\subsubsection{Application}

Fiber content and temperature are the main parameters that should be in better control of the production process in order to reduce the failure probability for tensile strength.

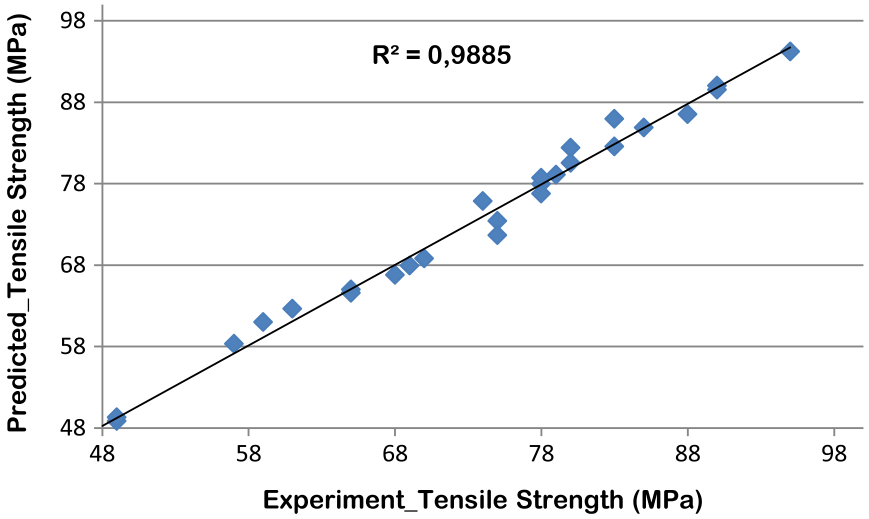

Fig. 3. Goodness of fit of RS model (Tensile strength).

The experimental results are coupled with the reliability-approach using the Response Surface Method. The basic idea of this method is to approximate the system response by an explicit function and to determine 


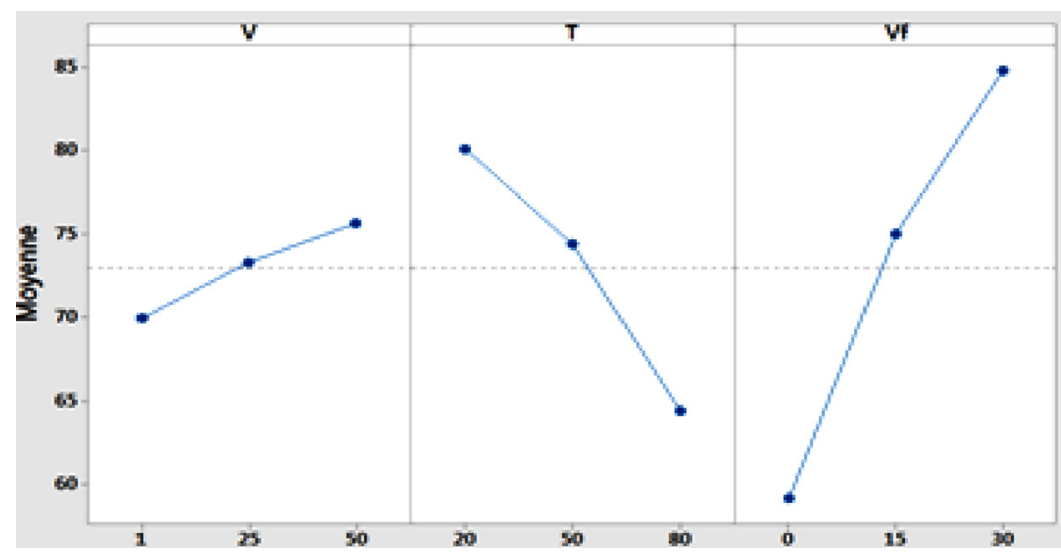

Fig. 4. Main effect plot of $\dot{\varepsilon}, T$ and $v_{f}$ on the value of the tensile strength.

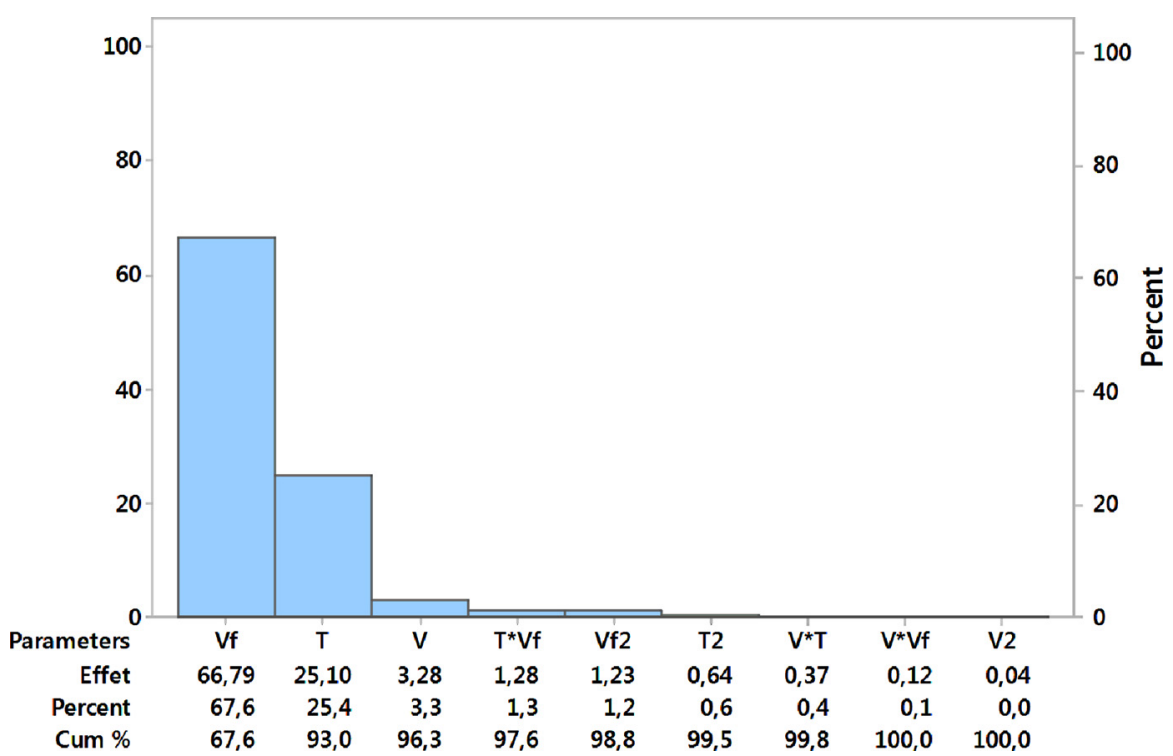

Fig. 5. Pareto chart of standardized effects on the tensile strength.

Table 3. Data characteristics.

\begin{tabular}{llll}
\hline Parameters & Law & Average & $\begin{array}{l}\text { Coefficient } \\
\text { of variation }\end{array}$ \\
\hline$v_{f}(\%)$ & Normal & 15 & $5 \%$ \\
$\boldsymbol{T}(\triangle \mathrm{C})$ & Normal & 50 & $5 \%$ \\
$\dot{\varepsilon}(\mathbf{m m} / \mathbf{m i n})$ & Deterministic & 25 & - \\
\hline
\end{tabular}

their distribution as a function of the required variables. The dispersion parameter is characterized by their coefficient of variation which is calculated as the ratio between the mean values and the standard deviation. The principle steps of computation the reliability is shown in the flowchart in Figure 2. MATLAB software is used to determine the distribution of tensile strength as a function of both temperature and fiber content. Then, the MCS is implemented for evaluating the failure probability, but before the computed reliabilities, it is necessary to optimize Monte Carlo sample size N. For the studied cases, the random parameters, their law and their characteristics are reported in Table 3. The variation of the reliability with used sample size is shown in Table 4 . It is observed that the discord between the computed reliabilities decreases with the Monte Carlo sample size $N$. When $N$ is higher than $10^{4}$, the percentage of the relative reliability variation value becomes less than $0.1 \%$. According to these results, the choice of the $N$ equal to $10^{4}$ is accepted [9].

Figures 6 and 7 illustrate the loading dispersion zones: case 1: tensile strength as a function of fiber content in which temperature is assumed to be normally distributed $(\mathrm{Cov}=5 \%)$, case 2: tensile strength as a function of temperature in which fiber content is assumed to be normally distributed $(\mathrm{Cov}=5 \%)$. 
Table 4. Sampling reliability computation $\left(\sigma_{r c}=58 \mathrm{MPa}, \operatorname{Cov} \sigma_{r c}=5 \%, v_{f}=0 \%, \operatorname{Cov} V_{f}=5 \%, \sigma_{r}=55 \mathrm{MPa}\right)$.

\begin{tabular}{llllllllllllll}
\hline & \multicolumn{1}{l}{ Reliabilities computation $(\%)$} & & & \\
Sample size $N$ & & R1 & R2 & R3 & R4 & R5 & R6 & R7 & R8 & R9 & R10 & Mean value & $E(r) \%$ \\
\cline { 2 - 10 } 1000 & 78.5 & 78 & 76.3 & 79.9 & 76.3 & 79.3 & 75.2 & 79.2 & 75.5 & 76 & 77.42 & 1.65 \\
5000 & 78.74 & 78.5 & 77.58 & 79.22 & 77.72 & 77.98 & 78.38 & 79.04 & 79.24 & 77.56 & 78.39 & 0.62 \\
10000 & 78.38 & 78.12 & 78.14 & 78.18 & 78.12 & 78.34 & 78.26 & 78.08 & 78.22 & 78.02 & 78.18 & 0.10 \\
20000 & 78.26 & 78.18 & 78.12 & 78.08 & 78.02 & 78.18 & 78.16 & 78.24 & 78.04 & 78.34 & 78.16 & 0.095 \\
30000 & 78.08 & 78.02 & 78.12 & 78.22 & 78.28 & 78.12 & 78.16 & 78.18 & 77.12 & 78.34 & 78.16 & 0.09 \\
\hline
\end{tabular}

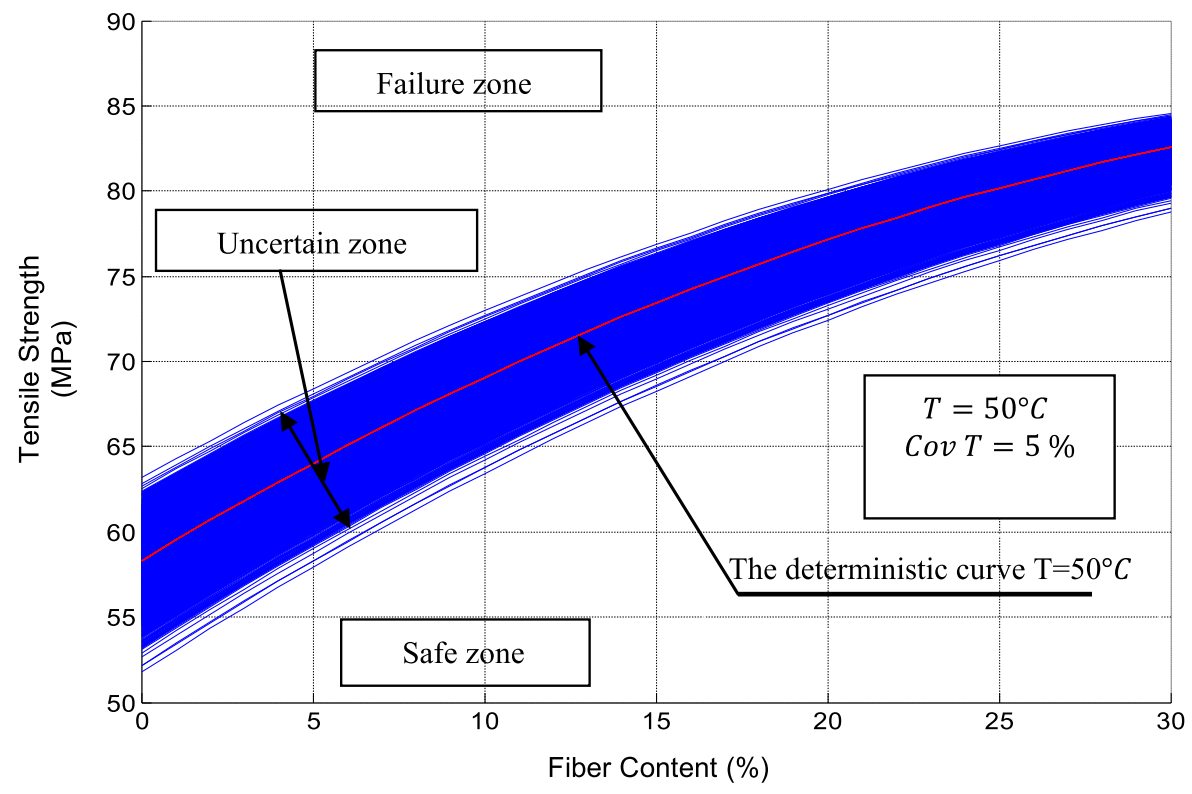

Fig. 6. Evolution of $\sigma_{r}$ versus fiber content with $\mathrm{Cov}=5 \%$.

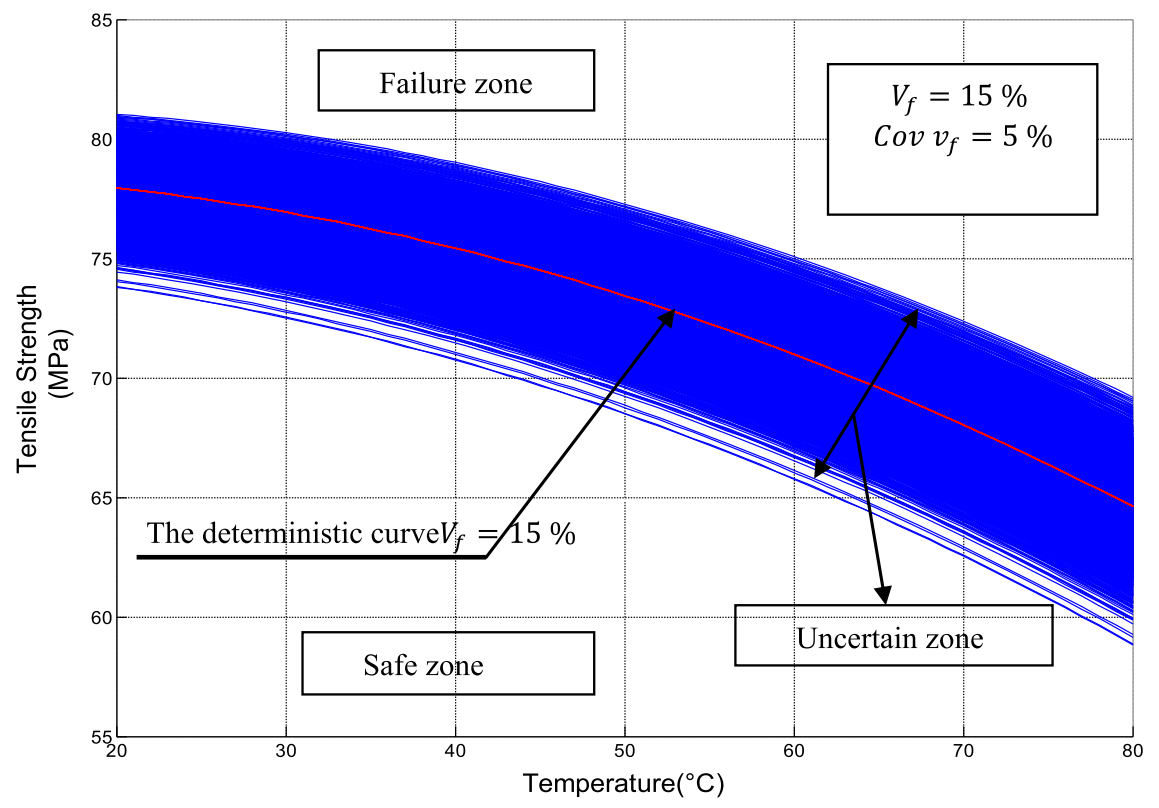

Fig. 7. Evolution of $\sigma_{r}$ versus temperature with $\mathrm{Cov}=5 \%$. 


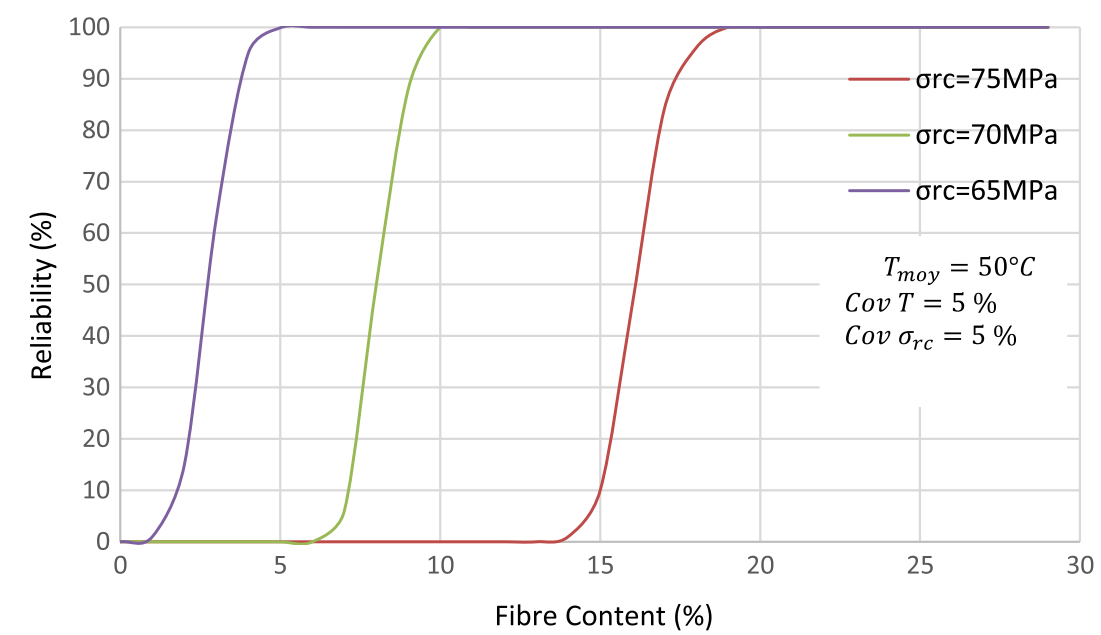

Fig. 8. Reliability versus Glass fiber content with different critical tensile strength.

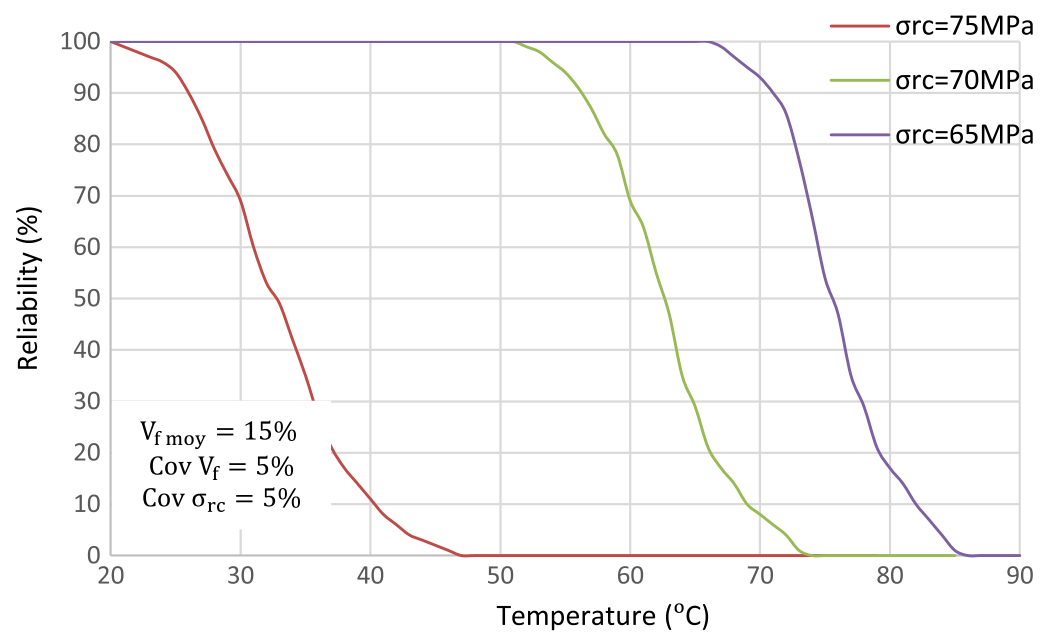

Fig. 9. Reliability versus Temperature with different critical tensile strength.

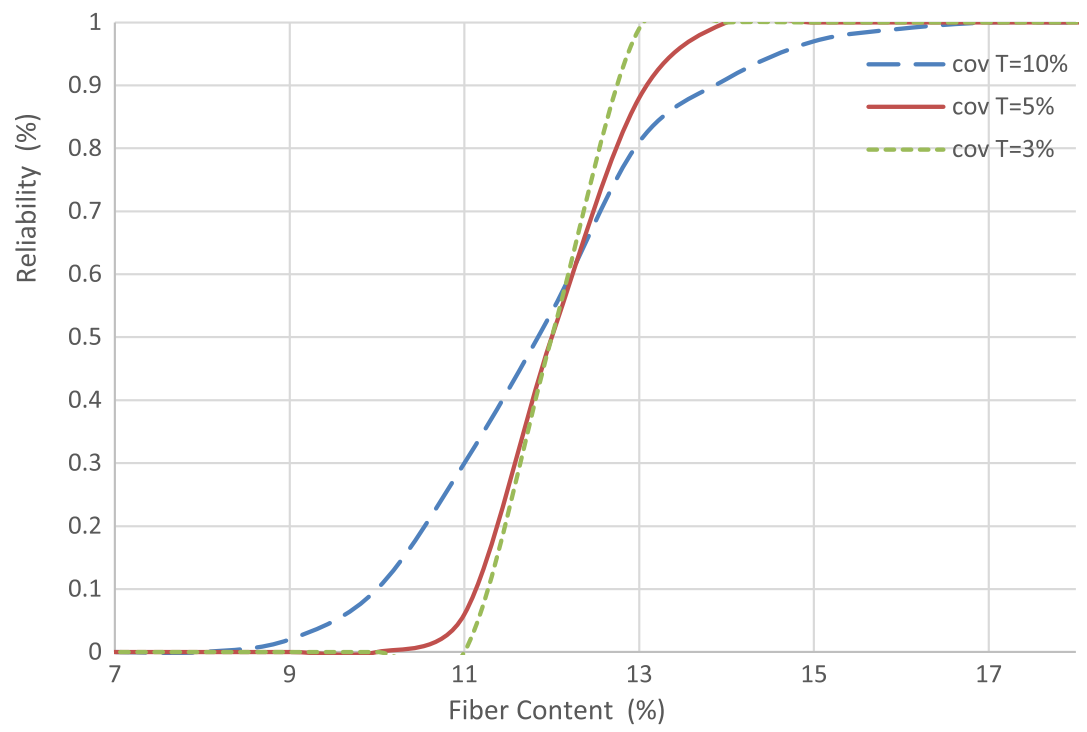

Fig. 10. Reliability versus fiber content with different Cov. 


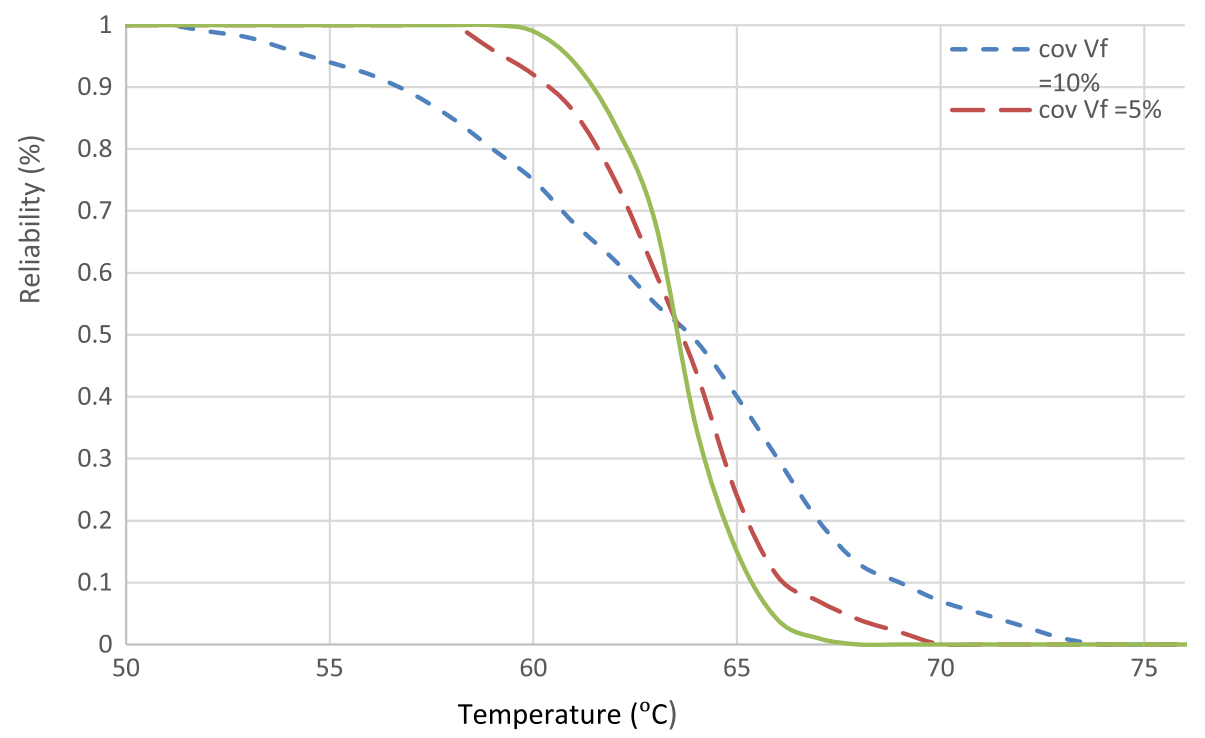

Fig. 11. Reliability versus Temperature with different Cov.

\subsubsection{Reliability results}

- From Figures 8 and 9, we can deduce the significant effect of the two factors $T$ and $v_{f}$ on the reliability index which is calculated using the MCS. It is interesting to note that the reliability decreases when the critical tensile strength increases.

we can conclude that the ratio of fiber has a very important effect on tensile strength such that if we add the fiber the reliability increases and is always in the field of safety. As a consequence, we can conclude that reliability decreases when critical tensile strength increases. For a reliability of $50 \%$, it is necessary to have $3 \%$ of the fiber content for $\sigma_{r c}$ equal to $65 \mathrm{MPa}$. On the other hand, it is necessary to have $16 \%$ of the fiber content for $\sigma_{r c}$ equal to $75 \mathrm{MPa}$.

Then, we note the non-linearity of the range between the extreme values of the temperature corresponding to $0 \%$ and $100 \%$ of the reliability. For a low value of temperature, it is noted that the reliability is equal to $100 \%$ against if the temperature exceeds $50^{\circ} \mathrm{C}$ (the glass transition temperature $\mathrm{Tg}$ of the polyamide 66 practically equal to $50^{\circ} \mathrm{C}$ ) the reliability decreases and tends to $0 \%$. These results are in good agreement with the experimental study [17], where it was observed that the change in the behavior of the polyamide occurs significantly in the vicinity of the glass transition temperature $(\mathrm{Tg})$ which is between 50 and $60^{\circ} \mathrm{C}$.

for safety condition of a glass fiber reinforced polyamide, the optimal parameters were obtained under the condition of $30 \%$ glass fiber content and $20{ }^{\circ} \mathrm{C}$ temperature.

- The developed probabilistic approach takes into account the dispersion of the fiber content and the temperature in which the tensile strength is considered as output parameters. In order to study the effect of different input parameters on the tensile strength of glass reinforced polyamide, we assume that all the probabilistic variables follow a normal distribution characterized by means and Coefficient of Variation $(\mathrm{CoV})$ values. Two parameters are considered as probabilistic design parameters with different $(\mathrm{CoV})$ values.

- Figures 10 and 11 presents the computed reliability curves plotted for the case of a $\mathrm{CoV}$ of $3 \%$, the case of a $\mathrm{CoV}$ of $5 \%$ and the case of $10 \%$ of fiber content and the temperature. It shows the dispersion of the $10^{4}$ design points for the three cases of study. For $v_{f}=13 \%$, the reliability $\mathrm{R}$ is approximated equal to $100 \%$ in the first case of study. For the second and third cases, the reliability decreases respectively to $92.3 \%$ and $70.7 \%$. Then for $T=60^{\circ} \mathrm{C}$, a reliability of $99.8 \%$ is estimates for the first case of study. For the second and third cases, the reliability decreases respectively to $97.1 \%$ and $86.7 \%$. It is observed that the scatter of the glass fiber content increases when the Cov value of the input temperature increases. As a consequence, we can observe a change of the range between the extreme values of the glass fiber content and the temperature, corresponding to the reliabilities of $0 \%$ and $100 \%$.

Finally, it can be concluded that the change in the coefficient of variation of the fiber content and temperature has a significant effect on the reliability of our composite material.

\section{Conclusions}

In this paper, a probabilistic approach based on the developed mathematical models for predicting the tensile strength reliability of a glass fiber reinforced polyamide is proposed. The Strength-Load method coupled with the MCS is implemented for evaluating the reliability with different conditions. The Response Surface Method (RSM) is used to calculate the performance function $G(\{X\})$ and their corresponding design points based on experimental results. An empirical relationship was developed based on the RSM approach for correlating the tensile strength with 
predominant process parameters. The basic idea of this method is to approximate the system response by an explicit function of random variables.

The following conclusions can be drawn:

- a probabilistic approach has been developed to evaluate the tensile strength reliability as a function of the temperature and fiber content. This approach leads to improve the deterministic models by taking into account the various dispersions of: (i) temperature and (ii) fiber content which are very significant and rarely considered. This observation clearly shows the capability of the probabilistic model to correctly take into account the statistical distribution of the input parameters in composite materials;

- based on the experimental observations and response surface methodology, we can investigate the tensile strength of our material with an analytical model. This result can be used also, to characterize qualitatively the effect of the different temperature and fiber content modifications. Moreover, we observe that an empirical relationship has been used to determine the damage threshold in the studied composites.

\section{Nomenclature}

$\begin{array}{ll}\sigma_{r} & \text { tensile strength (MPa) } \\ \sigma_{r c} & \text { critical tensile strength }(\mathrm{MPa}) \\ V_{f} & \text { fiber volume fraction }(\%) \\ T & \text { temperature }\left({ }^{\circ} \mathrm{C}\right) \\ \dot{\varepsilon} & \text { strain rate }(\mathrm{mm} / \mathrm{min}) \\ \{X\} & \text { vector of random variables } \\ \text { xi } & \text { element of the vector }\{X\} \\ f_{X i}(X i) & \text { probability density function of the variable xi } \\ \mathrm{RSM} & \text { Response Surface Methodology } \\ \mathrm{G}, \mathrm{S}, \mathrm{L} & \text { performance, strength and load functions, respec- } \\ & \text { tively } \\ P_{f} & \text { failure Probability } \\ \mathrm{FORM} & \text { first order reliability method } \\ \text { DoE } & \text { design of experiments } \\ \mu & \text { average value of the random variables } \\ \sigma & \text { standard deviation of the random variables } \\ \text { Cov } & \text { coefficient of variation (ratio of the standard } \\ N & \text { deviation of a distribution to its arithmetic mean) } \\ N & \text { Monte Carlo simulation sample size }\end{array}$

\section{References}

[1] M. Akay, D.F. Oregan, 'Fracture behaviour of glass fibre reinforced polyamide mouldings, Polym. Test. 14 (1995) 149-162

[2] J.P. Tancrez, J. Pabiot, F. Rietsch. Damage and fracture mechanisms in thermoplastic-matrix composites in relation to processing and structural parameters, Compos. Sci. Technol. 56 (1996) 725-731
[3] J.L. Thomason, The influence of fibre properties of the performance of glass-fibre-reinforced polyamide 66, Compos. Sci. Technol. 59 (1999) 2315-2328

[4] S.-Y. Fu, B. Lauke, A. San, Effect of fiber length and fiber orientation distributions on the tensile strength of short fiber reinforced polymers, Compos. Sci. Technol. 56 (1996) 1179 1190

[5] C. Bouraoui, R.B. Sghaier, R. Fathallah, An engineering predictive design approach of high cycle fatigue reliability of shot peened metallic parts, Mater. Des. 30 (2009) 475-486

[6] R.B. Sghaier, C. Bouraoui, R. Fathallah, T. Hassine, A. Dogui, Probabilistic high cycle fatigue behaviour prediction based on global approach criteria, Int. J. Fatigue 29 (2007) 209-221

[7] Q. Xueyong, R.T. Haftka, Deterministic and reliabilitybased optimization of composite laminates for cryogenic environments, AIAA J. 41 (2013) 10-19

[8] Z. Sun, C. Wang, X. Niu, Y. Song, A response surface approach for reliability analysis of $2.5 \mathrm{D} \mathrm{C} / \mathrm{SiC}$ composites turbine blade, Compos. Part B 85 (2016) 277-285

[9] M. Lemaire, A. Chateauneuf, J.C. Mitteau, Fiabilité des structures: couplage mécano-fiabiliste statique, Edit Hermes Paris 52 (2005) 620, [In French]

[10] H. Karadeniz, Uncertainty modeling in the fatigue reliability calculation of offshore structures, Reliab. Eng. Syst. Saf. 74 (2001) 23-35

[11] Y.G. Zhao, T. Ono, Oment for structural reliability, Struct. Saf. 23 (2001) 47-75

[12] P. Bjerager, Methods for structural reliability computation: Reliability problems: general principles and applications in mechanics of solid and structures, Springer Verlag, New York, Vol. 8, 1991, pp. 89-136

[13] A. Haldar, S. Mahadevan, Probability, reliability, and statistical methods in engineering design, John Wiley \& Sons, New York, 2000

[14] R. Rackwitz, Reliability analysis: a review and some perspectives, Struct. Saf. 23 (2001) 365-395

[15] R.H. Myers, D.H. Montgomery, Response surface methodology, John Wiley \& Sons, USA, 1995

[16] B. Mouhmid, A. Imad, N. Benseddiq, S. Benmedakhène and A. Maazouz, A study of the mechanical behaviour of a glass fibre reinforced polyamide 6, 6: Experimental investigation, Polym. Test. 25 (2006) 544-552

[17] B. Mouhmid, Etude de l'endommagement et de la rupture d'un polyamide 66 chargé en fibres de verre courtes, Thèse, Université des Sciences et Technologies de Lille, 2007

[18] V. Ceolho, Effects of strain rate and temperature on the mechanical properties of gfrp composites, Therm. Eng. 10 (2011) 03-06

[19] M. Todo, K. Takahashi, P. Beguelin, H. Kausch, Strain rate dependence of the tensile fracture behaviour of woven cloth reinforced polyamide composites, Compos. Sci. Technol. 60 (2000) 763-771

Cite this article as: M.M. Mhalla, A. Bahloul, C. Bouraoui, Reliability prediction of the tensile strength of a glass fiber reinforced polyamide using response surface method, Mechanics \& Industry 19, 110 (2018) 